\title{
Fournier's Gangrene without Organ Perforation Following Docetaxel: A Rare Urological Emergency Occurrence in a Patient with Metastatic Prostate Cancer
}

\author{
Ismail Selvi ${ }^{1 *}$, Fatih Hizli ${ }^{2}$ and Halil Basar ${ }^{2}$ \\ ${ }^{1}$ Department of Urology, Karabük University Training and Research Hospital, Karabük, Turkey \\ ${ }^{2}$ Department of Urology, Health Science University, Dr. Abdurrahman Yurtaslan Ankara Oncology Training and Research Hospital, Ankara, Turkey
}

*Corresponding author: Ismail Selvi, Department of Urology, Karabük University

\section{Abstract}

Fournier's gangrene (FG) which is known as necrotizing fasciitis of urogenital regions, is a rapidly progressive polybacterial infection, involving the subcutaneous and deep fascias in the urogenital region. The most common reasons are localized infections caused by colorectal, genitourinary and dermatological factors. On the other hand, its development following chemotherapy application is rare. Emergency and repetitive radical surgical debridement, wound drainage and parenteral antibiotherapy are mandatory because of high mortality rates in FG. In the literature, there is no reported case of FG in metastatic prostate cancer following docetaxel. We present a rare case of FG without visceral organ perforation, caused by docetaxel in a 72-year-old man. A similar case has not been reported. In literature, there are only a few known FG cases associated with bevacizumabrelated rectal and small intestinal perforation in rectal cancer.

Keywords: Docetaxel; Emergency surgical debridement; Fournier's gangrene; Metastatic prostate cancer

\section{Introduction}

Fournier's gangrene (FG) is a rapidly progressive acute disease of perineal, perianal or genital areas and it is a special name for necrotizing fasciitis caused by polymicrobial agents. While the most common reasons are localized infections caused by colorectal, genitourinary and dermatological factors, the others are genitourinary trauma, urologic instrumentation, presence of foreign bodies, recent surgery, inadequate perineal or urogenital hygiene and long term indwelling urethral catheters. On the other hand, its development following chemotherapy application is rare. Treatment consists of extensive surgical debridement, parenteral broad-spectrum antibiotherapy and reconstruction of defected areas $[1,2]$.

We present a case of severe FG in a patient with metastatic prostate cancer, following docetaxel administration. To our knowledge, there is no another example of case that indicates FG development without organ perforation, following docetaxel given for metastatic prostate cancer in the literature.

\section{Case Presentation}

A 72-year-old man with lower urinary tract symptoms was performed transrectal prostate biopsy four months ago due to PSA level was $64 \mathrm{ng} / \mathrm{ml}$. Biopsy was reported as prostate adenocarcinoma (Gleason $5+4$ ) and extensive bone metastasis were determined in the clinical evaluation. Androgen deprivation therapy (LHRH analogue) and concomitant chemotherapy (docetaxel) was planned because of diffuse bone involvement. After the second dose of docetaxel, the patient was presented with fever, swelling and severe pain in perineum and scrotum. His initial body temperature was $39.1{ }^{\circ} \mathrm{C}$, blood pressure was $90 / 65 \mathrm{mmHg}$, heart rate was 115 beats/min, and oxygen saturation was $94 \%$ on room air. On physical examination, erythema and tenderness in the inguinal region, lesions containing vesicles on the inguinal, perineal and scrotal skin and periurethral abscess were detected (Figure1). Leukocytosis $(15000 / \mu \mathrm{l})$, elevated hematocrit $(47.3 \%)$ and elevated C-reactive protein $(138 \mathrm{mg} / \mathrm{dl})$ were detected in 
laboratory tests. A necrotizing soft tissue infection with large amounts of gas throughout the perineum was revealed at ultrasound. Fournier gangrene severity index score was measured as 11 points. The patient had no other predisposing factors such as immunosuppressive systemic diseases, usage of chronic urethral catheter and other interventions to the urethra.

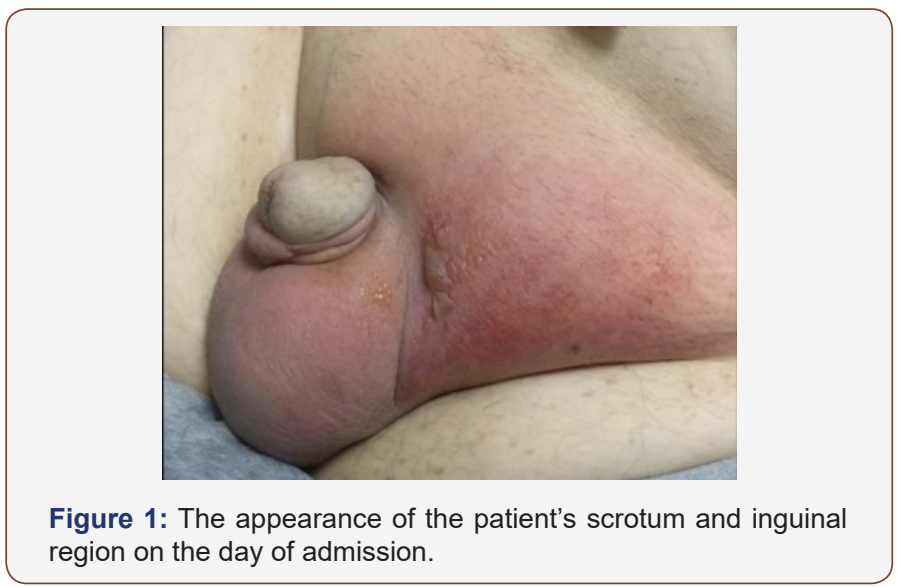

Since there was doubt about urethral involvement due to periurethral abscess, percutaneous cystostomy was placed to provide urinary drainage as an initial procedure. Daptomycin and meropenem were administered as intravenous antibiotherapy in consultation with an infectious disease specialist, following emergency surgical debridement which was performed at the inguinal region, perineum and scrotum. Because of testicular involvement with FG, bilateral orchiectomy was performed and percutaneous cystostomy was replaced by urethral catheter (Figure 2).

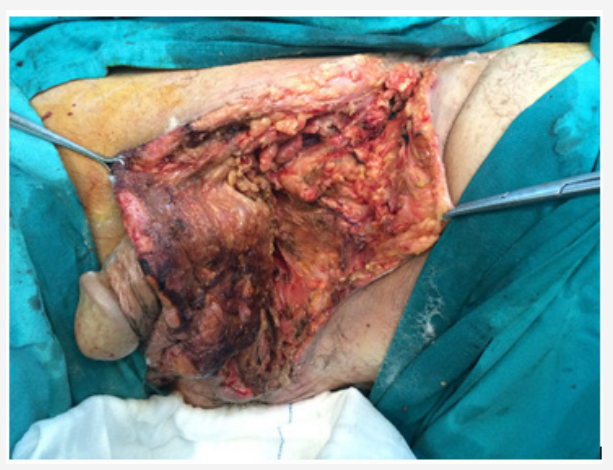

Figure 2: The appearance on the third day, following extensive surgical debridement and bilateral orchiectomy.

The debridement periods were repeated every two days until tenth day. The resultant defects following the debridement were covered with primary closure on the tenth day (Figure 3). We used solutions of Povidone-iodine, rifampin, gentamicin for daily wound dressings until primary closure. Escherichia coli, Enterococcus and anaerobes were detected in the cultures of perioperative tissues and current parenteral antibiotherapy was continued for two weeks. Histopathology of orchiectomy specimen was compatible with acute necrotizing inflammation. After two weeks, the patient was discharged with no complications, following nursing care and daily wound dressing.

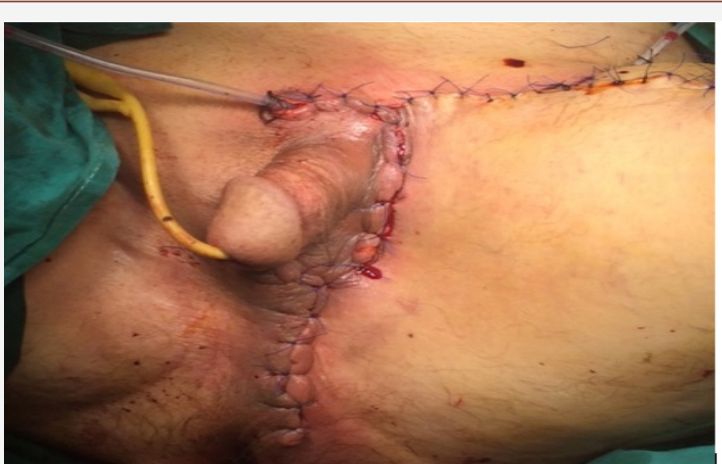

Figure 3: Defected tissues were covered with primary closure on the tenth day.

Since bilateral orchiectomy was performed, there was no need to continue the LHRH analogue as androgen deprivation therapy. Outpatient chemotherapy (docetaxel) was decided to continue, following discharge, after all wound has healed completely.

\section{Discussion}

Fournier's gangrene, originally described by Baurienne (1764) and Fournier (1883), is a rapidly fulminating gangrene in urogenital region [3]. Trauma and immunosuppressive conditions facilitate the access of infectious organisms to subcutaneous tissues. These organisms, which accompany the periurethral gland or perianal infections, extend along the retropubic space and facial planes. As a result, they invade penis, scrotum and perineum [4].

Urethral obstruction, urinary extravasation, urologic instrumentations, diabetes mellitus, alcoholism, immunosuppression, chronic debilitating illness, malignancy and chronic steroid usage take part in etiology of FG [5].

Inflammation and edema in the enclosed space cause impaired blood supply, hypoxia, so it facilitates acceleration of coagulation and is responsible for vascular thrombosis, subcutaneous tissue necrosis and dermal gangrene. In this way, facultative and obligatory anaerobes, such as E. coli, Bacteroides fragilis, and Clostridium perfringens easily grow [6].

FG is a urological emergency condition because it causes deaths due to sepsis and multiple organ failure. Therefore, Fournier's gangrene severity index (FGSI) has commonly been used for determining prognosis in recent years. The used predictions are body temperature, heart rate, respiratory rate, hematocrit, leukocyte count and serum levels of sodium, potassium, creatinine, bicarbonate $[4,7]$. According to this scale, a score of $>9$ is associated with a $75 \%$ mortality, while a score of $\leq 9$ is corresponded to a $78 \%$ probability of survival. Although an FGSI score of 11 points was observed in our patient, he was discharged without a major complication during his treatment. But he suffered organ loss due to testicular tissue involvement.

Because hyperbaric oxygen (HBO) is known as an inhibitor for the growth of anaerobic bacteria in the necrotic tissues, it can prevent further extension of tissue necrosis and reduce systemic toxicity. Therefore, HBO has been recently recommended as an 
additional therapy [8]. In our case, we did not perform HBO. Applying percutaneous cystostomy, early and repetitive radical debridement of necrotic tissues, wound drainage and parenteral antibiotherapy were adequately effective to manage the procedure.

Most of the FG occur without organ perforation. On the other hand, in the literature, visceral organ perforation is present in FG cases due to the use of immunosuppressive agents. For example, the literature indicates a few cases that small intestinal or rectal perforation during chemotherapy with bevacizumab and radiotherapy in patients with lower rectal cancer cause development of FG [9-11]. There are also a limited number of cases that have reported spontaneous rectal perforation, causing to FG in rectal cancer [12-14]. In these cases, we can see that the main cause of FG development is visceral organ perforation in rectal cancers due to chemotherapeutic agents except for docetaxel. Because intestinal perforation after treatment with bevacizumab is well known, the etiology in these cases can be explained with this pathogenesis. Since we could not find any other etiologic factors, we think that FG development in our patient is due to docetaxel as an immunosuppressant, based on information in the literature.

\section{Conclusion}

In conclusion, aggressive surgical debridement, broad-spectrum parenteral antibiotics, and intensive supportive care are critical for the management of FG. To our knowledge, there is no reported case that indicates FG development without organ perforation, following docetaxel given for metastatic prostate cancer in the literature. Although FG caused by docetaxel administration is very rare, it should be kept in mind as a differential diagnosis, when the patient has a known history of docetaxel usage.

\section{Informed Consent}

Written informed consent was obtained from patient who participated in this study.

\section{Acknowledgement}

None.

\section{Conflict of Interest}

Authors have no conflicts of interest to declare.

\section{References}

1. Paonam SS, Bag S (2015) Fournier gangrene with extensive necrosis of urethra and bladder mucosa: A rare occurrence in a patient with advanced prostate cancer. Urol Ann 7(4): 507-509.

2. Ameh EA, Dauda MM, Sabiu L, Mshelbwala PM, Mbibu HN, et al. (2004) Fournier's gangrene in neonates and infants. Eur J Pediatr Surg 14 (6): 418-421.

3. Schaffer AJ, Schaffer EM (2016) In: Infections of the urinary tract. In: Walsh C, Wein AJ, Kavoussi LR, Novisk AC, Partin AW, et al. (Eds.), Campbell's Urology. Philadelphia: Saunders, Elsevier pp: 302-303.

4. Doluoğlu ÖG, Karagöz MA, Kılınç MF, Karakan T, Yücetürk CN, et al. (2016) Overview of different scoring systems in Fournier's Gangrene and assessment of prognostic factors. Turk J Urol 42(3): 190-196.

5. Sorensen MD, Krieger JN (2016) Fournier's Gangrene: Epidemiology and Outcomes in the General US Population. Urol Int 97: 249-559.

6. Orhan E, Şenen D (2017) Using negative pressure therapy for improving skin graft taking on genital area defects following Fournier gangrene. Turk J Urol 43(3): 366-370.

7. Yoshino Y, Funahashi K, Okada R, Miura Y, Suzuki T, et al. (2016) Severe Fournier's gangrene in a patient with rectal cancer: case report and literature review. World J Surg Oncol 14(1): 234-238.

8. Mallikarjuna MN, Vijayakumar A, Partil VS, Shivswamy BS (2012) Fournier's gangrene: current practices. ISRN Surg.

9. Koyama M, Kitazawa M, Ehara T, Yamamoto Y, Suzuki A, et al. (2017) Two Cases of Fournier's Gangrene That Occurred during Chemotherapy for Rectal Cancer. Gan To Kagaku Ryoho 44(2): 169-171.

10. Fukuhisa H, Baba K, Kita Y, Tanabe H, Ijichi T, et al. (2017) A Case of Fournier's Gangrene Due to Perforation of Lower Rectal Cancer during Chemotherapy. Gan To Kagaku Ryoho 44(10): 935-937.

11. Ishida T, Shinozaki H, Ozawa H, Kobayashi T, Kato S, et al. (2016) A Case of Fournier's Gangrene Caused by Small Intestinal Perforation during Bevacizumab Combination Chemotherapy. Gan To Kagaku Ryoho 43(7): 909-911.

12. Sawayama H, Miyanari N, Sugihara H, Iwagami S, Mizumoto T, et al. (2016) A Case of Fournier's Gangrene Caused by Small Intestinal Perforation during Bevacizumab Combination Chemotherapy. Gan To Kagaku Ryoho 43(7): 909-911.

13. Ossibi PE, Souiki T, Ibn Majdoub K, Toughrai I, Laalim SA, et al. (2015) Fournier gangrene: rare complication of rectal cancer. Pan Afr Med J 20: 288-291.

14. Carr JA (2010) Perforated rectal cancer presenting as Fournier's gangrene. J Clin Oncol 28(30): e605-606. 